\title{
Enhanced Iris Recognition System - an Integrated Approach to Person Identification
}

\author{
Gaganpreet Kaur \\ Research Scholar, GNDEC, \\ Ludhiana.
}

\author{
Akshay Girdhar \\ Associate Professor, GNDEC. \\ Ludhiana.
}

\author{
Manvjeet Kaur \\ Lecturer, Punjab Engg.College, \\ Chandigarh.
}

\begin{abstract}
This paper discusses about Enhanced iris recognition which is used to overcome some of the problem like to automate the recognition of the iris by reducing complexity and increasing algorithm speed. Various challenges are faced while working with the iris recognition system. Iris recognition systems make use of the uniqueness of the iris patterns to derive a unique mapping. Iris recognition, as a biometric method, outperforms others because of its high accuracy. Iris recognition also has the ability to handle very large populations at high speed. Mostly three stages are followed while working with iris system i.e. preprocessing, feature extraction and recognition stage. This paper presents an automated and novel iris recognition system where overall computational match speed is reduced (from iris preprocessing to the final stage of recognition) and hence makes system more reliable with accuracy of $99.38 \%$ and low FAR.
\end{abstract}

\section{Keywords}

Biometrics, False Accept Rate (FAR), False Reject Rate (FRR), HD (Hamming Distance), CASIA (Chinese Academy of Sciences - Institute of Automation).

\section{INTRODUCTION}

The word "biometrics" is derived from the Greek words bio (life) and metric (to measure) [1]. It is general term used alternatively to describe a characteristic or a process. As a characteristic it is measurable biological and behavioral characteristic that can be used for automated recognition. As a process it encompasses automated methods of recognizing an individual based on measurable biological and behavioral characteristic. Biometric identification may be preferred over traditional methods (e.g. passwords, smart-cards) because its information is virtually impossible to steal. Iris recognition has a very good balance of all the properties. A number of biometric characteristics are being used in various applications as Universality, Uniqueness, Permanence, Measurability, Performance, Acceptability, and Circumvention [2].

\subsection{Iris}

An iris is internal organ of eye that is well protected against damage and wear by a highly transparent and sensitive membrane (the cornea). Figure 1 shows an eye image with different portions of eye [3]. It is believed with strong evidences that each iris is unique. Each person has his own iris with the permanent uniqueness. So iris has being used for identification and forensic investigation.

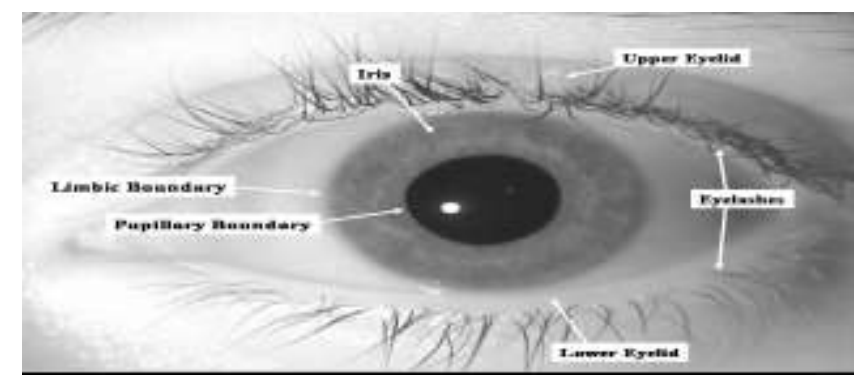

Figure 1: An eye image illustrating different portions of eye

\subsection{Iris Recognition}

"Iris recognition" is a method of biometric authentication that uses pattern recognition techniques based on high-resolution images of the irides of an individual's eyes. Iris systems have a very low False Accept Rate (FAR) compared to other biometric traits; the False Reject Rate (FRR) of these systems can be rather high [2]. Iris recognition analyzes features like rings, furrows, and freckles existing in the colored tissue surrounding the pupil. Image processing techniques can be employed to extract the unique iris pattern from a digitized image of the eye, and encode it into a biometric template, which can be stored in a database. This biometric template contains an objective mathematical representation of the unique information stored in the iris, and allows comparisons to be made between templates.

\subsection{Iris Recognition System}

A typical iris recognition system as shown in Figure 2 involves three main modules:

\subsubsection{Image acquisition}

It is to capture a sequence of iris images from the subject using a specifically designed sensor.

\subsubsection{Preprocessing Stage}

It includes determining the boundary of the iris within the eye image, and extracts the iris portion from the image to facilitate its processing. It includes various stages such as:

a. Iris Segmentation

b. Iris localization and Algorithm

c. Eyelash and Noise Detection

d. Iris Normalization

e. Image Enhancement 


\subsubsection{Feature extraction and Encoding}

This is the most key component of an iris recognition system and determines the system's performance to a large extent. Iris recognition produces the correct result by extracting features of the input images and matching these features with known patterns in the feature database.

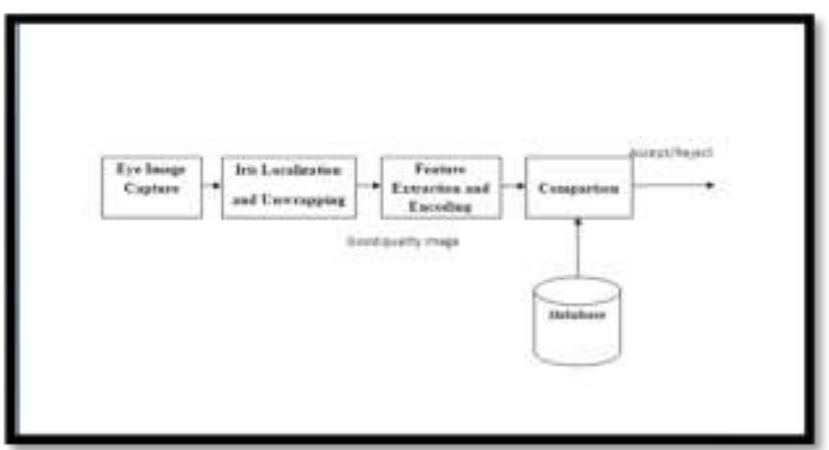

Figure 2: Iris Recognition System

\section{DESIGN AND IMPLEMENTATION OF ENHANCED IRIS RECOGNITION SYSTEM}

In this work the major focus is to implement the iris recognition system that provides reliability, accuracy and reduced overall match speed.

\subsection{Proposed Model}

From the literature, it has been found that there is a need to develop a new iris system in which overall system operation time can be reduced. In iris localization, only available existing techniques like Hough Transform and Integero-differential operator method are used. The proposed model focuses on following steps which are helpful in decreasing the computational match time:

a. Computational time of match and overall processing can be simplified.

b. Low FAR and FRR should be achieved.

c. To compare the enhanced system with other technique.

This is practically implemented using MATLAB 7.0 environment.

\subsection{System Level Design}

An iris recognition system constitutes of Iris Acquiring device (sensor) for generating digital image of iris. For iris acquisition, optical or semi-conduct sensors are widely used. They have high efficiency and acceptable accuracy except for some cases that the user's eye is too far. In this work, the testing database for iris provided by The Chinese Academy of Sciences - Institute of Automation (CASIA-IrisV3). As digital image of iris is available, so no acquisition stage is implemented. Figure 3 shows the test images used in Enhanced Iris Recognition System.

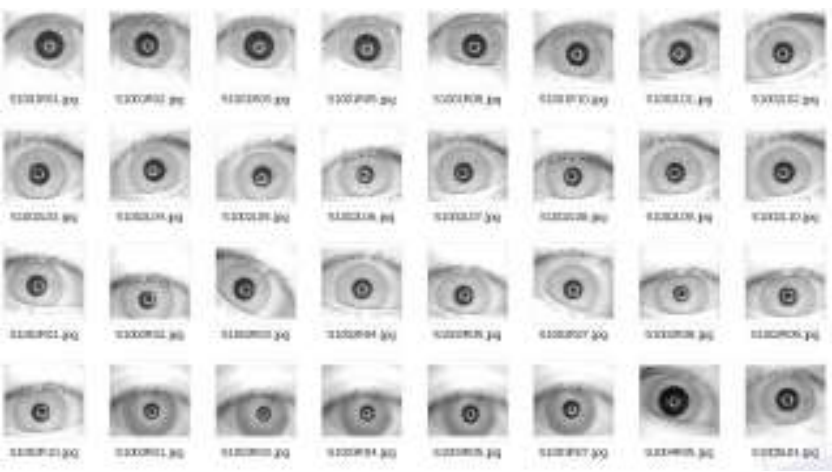

Figure 3: Iris Test Images

\subsection{Algorithm Level Design}

To implement iris recognition, a three-stage approach is widely used. These stages are preprocessing, feature extraction and recognition stage. The steps of the complete process used in the work are shown in Figure 4.

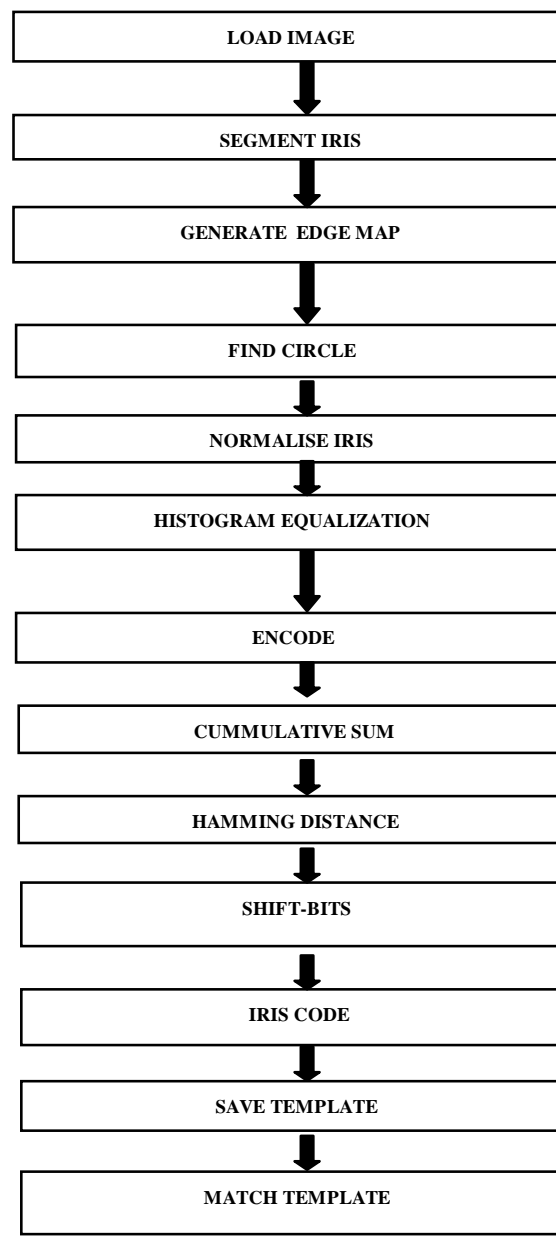

PREPROCESSING IRIS FEATURE

RECOGNITION 


\subsubsection{Iris Image Preprocessing}

Following are the various steps during image preprocessing stage:

1) Iris Segmentation: This involves first employing Canny Edge Detection to generate an edge map.

2) Iris Localization: In the work, in order to increase the overall speed of the system, circle detection algorithm [4] is used.

Circle detection in the work contributes to:

a. It has good recognition performance and speed.

b. The algorithm is able to very accurately detect even partially occluded circles.

c. The algorithm needs a very small amount of memory.

d. The algorithm creates low processing burden than other methods.

e. It is simple and efficient method.

3) Iris Normalisation: After successfully extracting the iris part from the eye image, in order to allow comparisons between different irises, transform the extracted iris region so that it has a fixed dimension, and hence removing the dimensional inconsistencies between eye images due to the stretching of the iris caused by the pupil dilation from varying levels of illumination. Therefore, this normalization process will produce irises with same fixed dimensions so that two photographs for the same iris under different lighting conditions will have the same characteristic features.

4) Iris Image Enhancement and Denoising: The iris image is enhanced by means of local histogram equalization and removes high-frequency noise by filtering the image with a low-pass Gaussian filter.

\subsubsection{Iris Feature Extraction and Encoding}

Cumulative Sum Based Analysis Method [5] is used to extract features from iris images. It analyzes the grey values patterns in iris image and hence extracts iris features. Steps are:

a) The iris image is divided into $m * n$ pixels cell regions.

b) Then five basic cell regions are grouped and cumulative sum is calculated.

c) Then iris codes are generated, if the value of cumulative sum is on upward slope then cell's iris code to 1 (i.e. darkness to brightness) and if on downward slope to 2 else to 0 .

The $\mathrm{m}^{*} \mathrm{n}$ value for Horizontal and Vertical direction is taken as $4 * 4$ pixels in cumulative sum based method for feature extraction. The minimum and maximum value for horizontal and vertical direction is taken as 0 and 255 in iris code generation algorithm.

\subsubsection{Iris Feature Matching}

To calculate the similarity of two iris codes, Hamming Distance (HD) method is used as given in 1. Lower Hamming Distance means the higher similarity [8].

$\mathrm{HD}=\frac{1}{\mathbb{N}}\left[\left(\Sigma_{i=1}^{\mathscr{W}} A_{\hbar}(i) \oplus B_{h}(i)\right)+\left(\Sigma_{\bar{i}=1}^{\mathscr{H}} A_{v}(i) \bar{\oplus} B_{v}(i)\right)\right](1)$
Here $A_{h}(\mathrm{i})$ and $A_{W}$ (i) mean enrolled iris codes over the horizontal and vertical direction and $\mathbb{B}_{\mathfrak{h}}$ (i) and $\mathbb{B}_{\mathbb{D}}$ (i) mean new input iris codes over the horizontal and vertical direction.

\section{RESULTS}

The performance of Enhanced Iris Recognition System is compared with Existing Iris Recognition System [6] in terms of computational speed and reliability. The overall computational time taken by system is reduced to a greater level. 141 genuine and 111 impostor user iris images have been provided to the system to calculate FAR and FRR values, out of which at Hamming Distance 0.39, 87 iris images were falsely rejected and 1 iris image was falsely accepted in enhanced iris recognition system.

\subsection{Performance Evaluation}

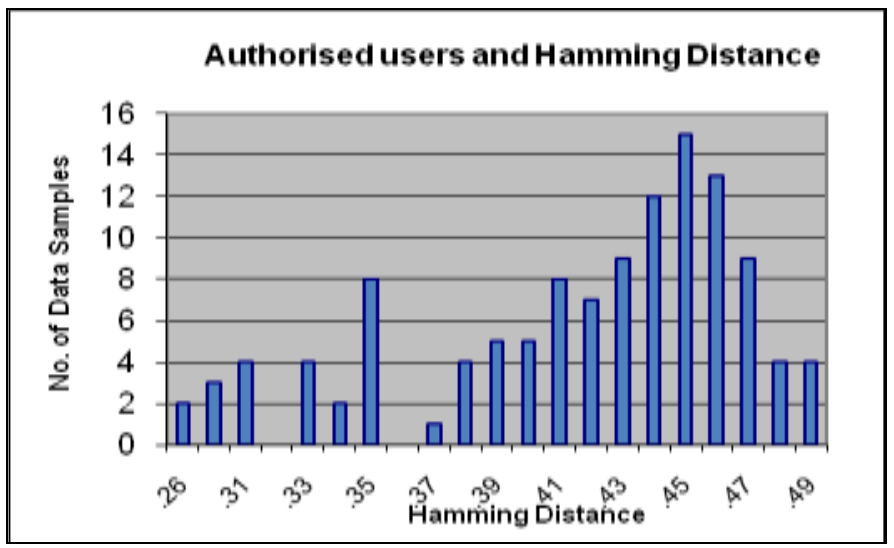

Figure 4: Graph showing Hamming Distance for the same persons (Authentic) for Enhanced Iris Recognition System.

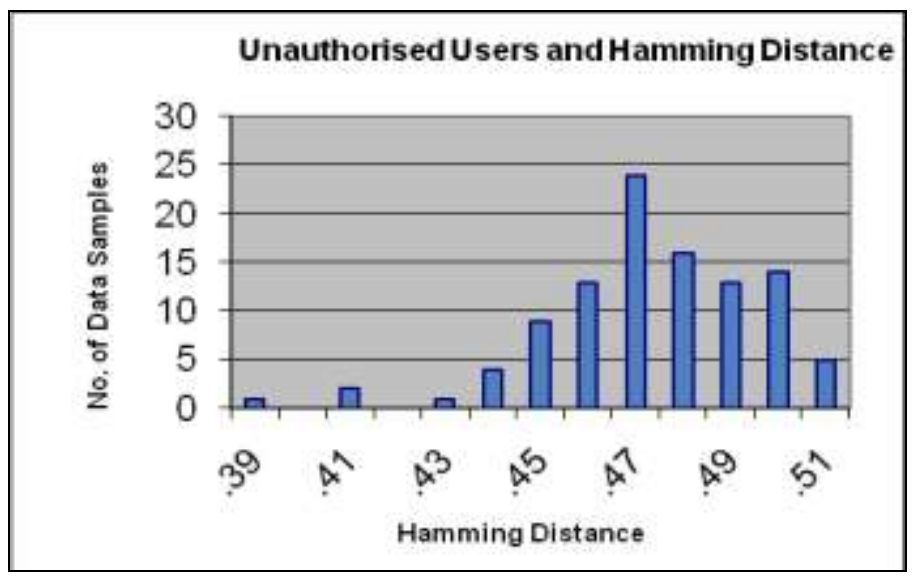

Figure 5: Graph showing Hamming Distance for the different persons (Impostors) for Enhanced Iris Recognition System. 


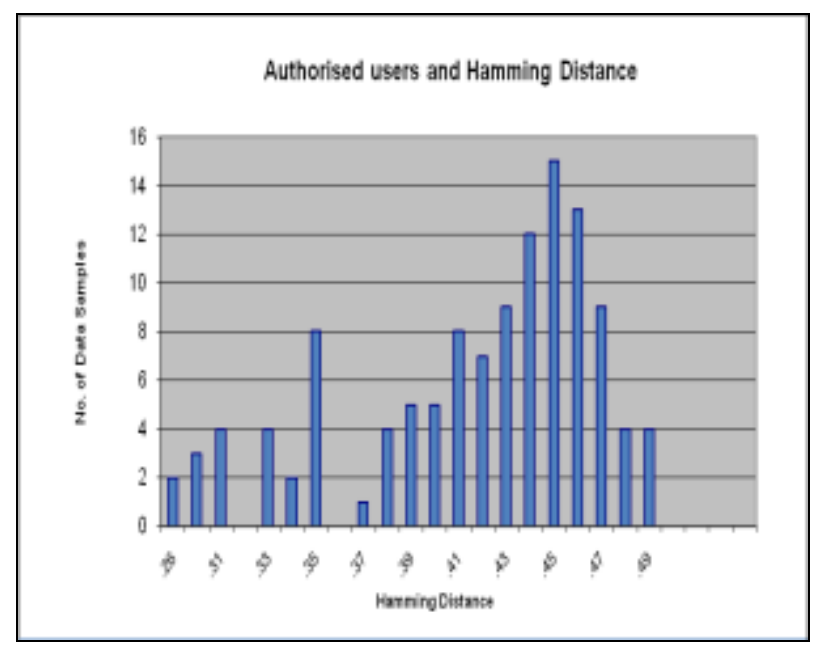

Figure 6: Graph showing Hamming Distance for the same persons (Authentic) for Existing Iris Recognition System.

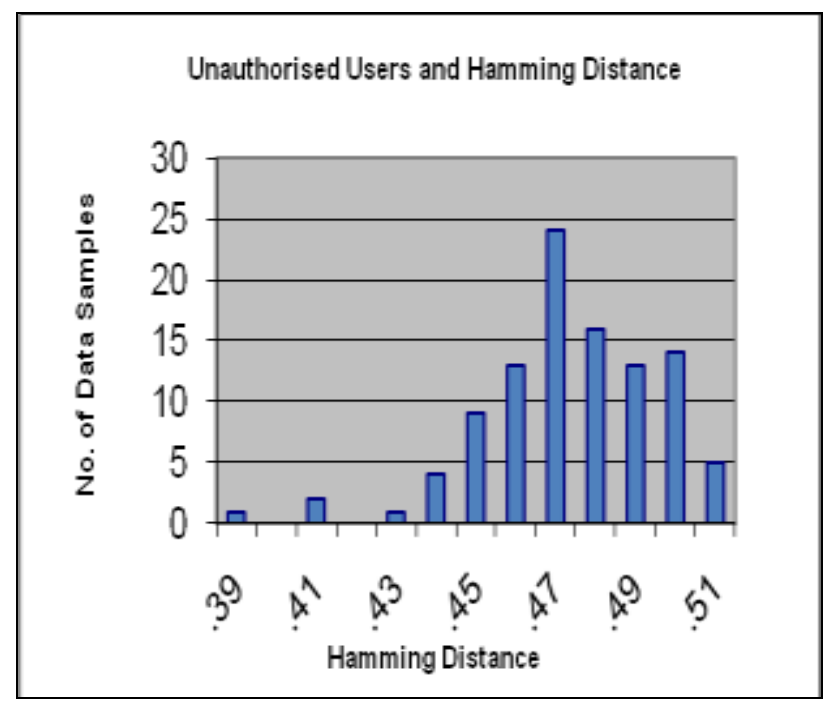

Figure 7: Graph showing Hamming Distance for the different persons (Impostors) for Existing Iris Recognition System.

Figure 4 and 5 shows hamming distance of authentic and impostors users for enhanced iris recognition system. $\mathrm{X}$-axis and Y-axis indicate the hamming distance and number of data samples. Hamming distance values for authorized users are distributed from .26 to .49 approx. and values for intruders are distributed from .39 to .51 .Figure 6 and 7 shows hamming distance of authentic and impostors users for existing iris recognition system.
Table 1: Comparison of the results of the Accuracy of Enhanced and Existing Iris Recognition System.

\begin{tabular}{|c|c|}
\hline IRIS RECOGNITION SYSTEM & ACCURACY \\
\hline Enhanced Iris Recognition & 99.38 with FAR/FRR $=$ \\
System & $0.01 / 0.61$ \\
\hline Existing Iris Recognition System & 99.37 with FAR/FRR = \\
& $0.05 / 0.58$ \\
\hline
\end{tabular}

\subsection{Computational Complexity}

Table 2: Comparison of Calculated Total Computational Time

\begin{tabular}{|c|c|c|c|c|c|c|}
\hline $\begin{array}{l}\text { METH } \\
\text { ODS }\end{array}$ & \multicolumn{6}{|c|}{$\begin{array}{l}\text { COMPUTATIONAL TIME (sec.) } \\
\text { (For iris image) }\end{array}$} \\
\hline $\begin{array}{l}\text { Iris } \rightarrow \\
\text { Images }\end{array}$ & $\begin{array}{l}\text { S100 } \\
\text { 1R01 }\end{array}$ & $\begin{array}{l}\mathrm{S} 100 \\
1 \mathrm{R} 03\end{array}$ & $\begin{array}{l}\text { S100 } \\
\text { 1R05 }\end{array}$ & $\begin{array}{l}\text { S100 } \\
\text { 2R01 }\end{array}$ & $\begin{array}{l}\mathrm{S} 100 \\
1 \mathrm{R} 10\end{array}$ & S1002L01 \\
\hline $\begin{array}{l}\text { Existin } \\
\mathrm{g} \quad \text { Iris } \\
\text { Recogn } \\
\text { ition } \\
\text { System }\end{array}$ & 7.672 & 9.438 & 8.219 & 7.734 & 5.953 & 7.016 \\
\hline $\begin{array}{l}\text { Enhanc } \\
\text { ed Iris } \\
\text { Recogn } \\
\text { ition } \\
\text { System }\end{array}$ & 0.953 & 0.984 & 0.922 & 1.016 & 1.109 & 1.109 \\
\hline
\end{tabular}




\section{CONCLUSION AND FUTURE SCOPE}

Experimental results show that in the Enhanced Iris Recognition system the overall computational match speed is reduced and makes system more reliable with accuracy of $99.38 \%$.

For the future work the image segmentation techniques or noise removal methods can be improved, so that the input image to the feature extraction stage could be made better which can improve the final outcome. Also, the system should be tested on a larger database to validate the robustness of the system.

\section{REFERENCES}

[1] Bhalchandra, A., Deshpande, N., Pantawane, N. and Kharwandikar, P. (2008), "Iris Recognition", Proceedings Of World Academy Of Science, Engineering And Technology, Vol. 36, pp. 1073-1078.

[2] Jain, A., Flynn, P. and Ross, A. (2008), "Handbook of Biometrics", Michigan State University, USA, Flynn University of Notre Dame, USA, West Virginia University, USA, pp. 79-98.

[3] Elsherief, S., Allam, M. and Fakhr, M. (2006), "Biometric Personal Identification Based on Iris Recognition" IEEE, pp. 208-213.

[4] Wu, J., Li, J., Xiao, C., Tan, F. and Gu, C. (2008), "Realtime Robust Algorithm for Circle Object Detection", Proceedings of the 9th International Conference for Young Computer Scientists, pp. 1722-1727.
[5] Ko, J., Gil, Y. and Yoo, J. (2006), "Iris Recognition using Cumulative SUM based Change Analysis", International Symposium on Intelligent Signal Processing and Communication Systems, Yonago Convention Center, Tottori, Japan, pp. 275-278.

[6] Ma, L., Tan, T., Wang, Y. and Zhang, D. (2003), "Personal Identification Based on Iris Texture Analysis", IEEE Trans. Pattern Anal. Mach. Intell., Vol. 25 No.12 pp. 1519-1533.

[7] Daugman, J. (2004), "How Iris Recognition Works", IEEE Transactions on Circuits and Systems for Video Technology, Vol. 14, No. 1 pp. 21-30.

[8] Masek, L. (2003), "Recognition of Human Iris Patterns For Biometric identification", Thesis Report, The University of Western Australia.

[9] Bowyer, K., Hollingsworth, K., and Flynn, P. (2007), "Image Understanding for Iris Biometrics: A survey", Elsevier, Computer Vision and Image Understanding, pp. 281-307.

[10] Daugman, J. (2007), "New Methods in Iris Recognition", IEEE Transactions on Systems, Man, and Cybernetics-Part B: Cybernetics, Vol. 37, No. 5 pp. 1167-1175.

[11] Wildes, R. (1997), "Iris Recognition, An Emerging. Biometric Technology", Proceedings of IEEE, Vol. 85, No. 9 pp. $1348-1363$. 